\title{
Lectura de gráficos estadísticos por profesores de educación primaria en activo
}

\author{
Danilo Díaz-Levicoy,3, José H. Parra-Fica², María Aravena-Díaz ${ }^{1,3}$ y Ximena Gutiérrez-Saldivia ${ }^{4}$ \\ (1) Facultad de Ciencias Básicas, Universidad Católica del Maule, Avda. San Miguel 3605, Talca-Chile \\ (2) Facultad de Ciencias de la Educación, Universidad Católica del Maule, Avda. San Miguel 3605, Talca-Chile \\ (3) Centro de Investigación en Educación Matemática y Estadística, Universidad Católica del Maule, Avda. San Miguel \\ 3605, Talca-Chile. \\ (4) Facultad de Educación, Universidad Católica del Temuco, Manuel Montt 56, Temuco-Chile \\ (Correo-e: dddiaz01@hotmail.com; jparra@ucm.cl; maravenadiaz@gmail.com; xgutierrez.saldivia@gmail.com)
}

Recibido Oct. 8, 2020; Aceptado Dic. 21, 2020; Versión final Feb. 8, 2021, Publicado Jun. 2021

\begin{abstract}
Resumen
El objetivo de esta investigación es evaluar la calidad y el nivel de lectura que alcanzan profesores de educación primaria en activo sobre gráficos estadísticos. La investigación es del tipo mixta, de nivel descriptivo y utiliza como método el análisis de contenido. La muestra del estudio es intencional con 21 participantes, 19 mujeres y 2 hombres, y con una media de 11.2 años de experiencia docente. Se aplicó un instrumento con tres ítems relacionados con la lectura de gráficos estadísticos. Como resultado se observa que los participantes presentan errores de cuantificación y cálculo, desconocimiento de los elementos constituyentes del gráfico y su lectura, y poseen una escasa capacidad argumentativa para explicar el efecto visual que produce la modificación de una escala. Se concluye que este grupo de profesores en activo presentan desconocimiento de algunos contenidos y habilidades sobre gráficos estadísticos, temática con amplia presencia en el currículo y que deben enseñar.
\end{abstract}

Palabras clave: gráficos estadísticos; nivel de lectura; calidad de lectura; educación primaria

\section{Reading of statistical graphs by primary education teachers}

\begin{abstract}
The aim of this study was to evaluate the quality and level of statistical graph reading skills of elementary school teachers. This study used a mixed approach, was descriptive, and the content analysis method was applied. The sample included 21 participants, 19 women and 2 men. The teaching experience mean was equal to 11.2 years. An instrument with three items related to the reading of statistical graphs was applied. The teachers surveyed showed quantification and calculation errors, poor understanding of graph core components, difficulties reading and analyzing graphs, and limited skills to explain the visual effects produced by scale modifications. In conclusion, the elementary school teachers surveyed lacked both knowledge of statistical graph core components and skills to analyze statistical graphs, which is a common and recurrent subject in the primary education curriculum.
\end{abstract}




\section{INTRODUCCIÓN}

En la actualidad, los ciudadanos tienen acceso a información estadística proveniente de diferentes fuentes, entre ellos los medios de comunicación (Gal, 2003, 2011; Koetsenruijter, 2011; McConway, 2016; Monteiro y Ainley, 2007), donde aparecen con frecuencia gráficos, tablas y resúmenes estadísticos (del Pino y Estrella, 2012; Díaz-Levicoy et al., 2019; Jurečková y Csachová, 2020). Estos elementos son considerados parte de la cultura estadística (Arteaga et al., 2016; del Pino y Estrella, 2012; Gal, 2002), la que hace referencia a un derecho ciudadano. De acuerdo con del Pino y Estrella (2012), la cultura estadística implica, entre otras habilidades, la lectura de representaciones estadísticas (tablas y gráficos estadísticos) y medidas de resumen que se muestras en los medios de comunicación, así como la valoración de la estadística en la vida cotidiana, cívica y profesional.

Dada esta importancia de la estadística, es que sus contenidos son incorporados en las directrices curriculares de Educación Primaria de diferentes países en la asignatura de matemática (Callingham y Watson, 2017; Díaz-Levicoy et al., 2019; Estrella et al., 2015), lo que también ha motivado el desarrollo de investigaciones en el área, por ejemplo, del conocimiento que tienen los profesores para la enseñanza de estas temáticas. Chile no ha quedado ajeno a esta tendencia y, en la última modificación realizada al currículo nacional (MINEDUC, 2018), considera la enseñanza de la estadística y la probabilidad desde los primeros años de Educación Primaria. Las actuales orientaciones curriculares dividen la enseñanza de la matemática en cinco ejes, incluyéndose el estudio de los gráficos estadísticos en el eje que se denomina Datos y Probabilidades. Este eje pretende, entre otras cosas, que "todos los estudiantes registren, clasifiquen y lean información dispuesta en tablas y gráficos, y que se inicien en temas relacionados con la probabilidad. Estos conocimientos les permitirán reconocer gráficos y tablas en su vida cotidiana" (MINEDUC, 2018). Este eje establece el estudio de los siguientes tipos de gráficos estadísticos: pictogramas ( $1^{\circ}$ a $4^{\circ} \mathrm{curso}$ ), gráficos de barras simples, con escala o barras dobles ( $2^{\circ}$ a $6^{\circ}$ curso), diagramas de puntos ( $3^{\circ}$ y $6^{\circ}$ curso), gráficos de línea ( $\left.5^{\circ} \mathrm{curso}\right)$, diagramas de tallo y hojas $\left(5^{\circ}\right.$ y $6^{\circ}$ curso) y gráficos de sectores ( $\left.6^{\circ} \mathrm{curso}\right)$. Además, se deben abordar aspectos sobre recolección, clasificación y registro de datos provenientes de contextos significativos.

Estas modificaciones curriculares han impactado en la formación de profesores, debido a que estos temas pasan a constituir parte importante del conocimiento disciplinar que deben poseer al egresar de su formación inicial. Cabe destacar que el MINEDUC (2012), a través del Centro de Perfeccionamiento, Experimentación e Investigaciones Pedagógicas (CPEIP), estableció en los Estándares orientadores para egresados de carreras de pedagogía en Educación Básica, los conocimientos, habilidades pedagógicas y disciplinares que debe poseer todo profesor de Educación Primaria al egresar de algún programa de formación pedagógica en Chile. Estos estándares se clasifican en dos categorías: pedagógicos y disciplinares. Los pedagógicos están compuestos por diez estándares, que corresponden a los conocimientos y habilidades que debe poseer todo egresado de pedagogía, independiente de su especialidad. Por otro lado, los disciplinares asociados con la matemática se dividen en cuatro ejes: 1) números; 2) geometría; 3) álgebra; 4) datos y probabilidades. Este último indica lo que debe saber todo profesor de Educación Primaria para la enseñanza de la estadística y probabilidad, así como las diferentes representaciones estadísticas consideradas en el currículo de Educación Primaria, resumido en los siguientes estándares (MINEDUC, 2012): 1) Es capaz de conducir el aprendizaje de la recolección y análisis de datos; 2) Demuestra competencia disciplinaria en el eje de Datos y Probabilidades.

Producto de estos cambios, diversos investigadores han centrado su interés en la lectura de gráficos estadísticos en profesores de diferentes niveles de formación. Entre ellos, Monteiro y Ainley (2007) quienes estudian la comprensión de gráficos tomados desde la prensa por futuros profesores brasileños. Los resultados dan cuenta que una cantidad importante de profesores en formación no tenían los conocimientos matemáticos necesarios para realizar dicha lectura. Además, reportan que solo algunos profesores en formación logran interpretar adecuadamente el gráfico estadístico y el significado en el contexto de la noticia.

Espinel et al. (2008) analizan la lectura de histogramas que hacen 137 futuros profesores de Educación Primaria. Entre los resultados observan la confusión entre el histograma y el diagrama de barras, además de la dificultad para identificar el gráfico estadístico más adecuado de acuerdo con la naturaleza de los datos. Rodríguez-Alveal y Sandoval (2012) indagan sobre la lectura de gráficos estadísticos (barras simples, barras dobles y sectores) en profesores de Educación Primaria (44 en formación y 47 en activo) en Chile. Sus resultados muestran que el $90 \%$ de los estudiantes de pedagogía y profesores en activo realizan alguna lectura de estas representaciones, el $79 \%$ lo hace de forma correcta, resultado más sencillo la lectura del gráfico de barras simples y más complejo el de barras agrupadas.

Más recientemente, Rodríguez-Alveal y Díaz-Levicoy (2019) analizan las habilidades de lectura de información cuantitativa unidimensional resumida en histogramas y polígonos de frecuencias simples, e información bidimensional presentada mediante polígonos de frecuencias simples, histogramas y gráficos de 
barras. Para ello, aplicaron un cuestionario a 61 participantes (30 profesores en activo y 31 profesores en formación) de una universidad de la zona central de Chile. Los resultados, novedosos en el contexto chileno, muestran que los profesores en formación y activos presentan porcentajes altos en la habilidad de lectura básica de información de un gráfico de barras, mientras que estos valores disminuyen al $61,3 \%$ (futuros profesores) y al $50 \%$ (profesores en activo) al leer un histograma. Los autores concluyen que, en general, los profesores participantes presentan dificultades para la lectura e interpretación de representaciones gráficas y no siempre son capaces de distinguir los elementos clave resumidas en ellas. Pese a estos estudios, la lectura y construcción de gráficos estadísticos sigue siendo un tema poco estudiado en Chile. De esta forma y de acuerdo con los antecedentes descritos, el presente estudio tiene como objetivo evaluar la calidad y el nivel de lectura que alcanzan profesores de Educación Primaria en activo sobre gráficos estadísticos.

Debido a los diversos elementos que intervienen en la lectura y construcción de gráficos estadísticos, estas actividades semióticas se consideran complejas, y han sido caracterizadas por diversos autores. Entre ellos, Curcio y cols. (Curcio, 1987; Friel, Curcio y Bright, 2001; Shaughnessy et al., 1996), proponen cuatro niveles de lectura: i) Nivel 1. Leer los datos. Cuando se demanda la lectura directa o literal de algún elemento del gráfico estadístico. Por ejemplo, identificar el título o leer alguna de las variables representada en el gráfico; ii) Nivel 2. Leer dentro de los datos. Cuando se pide realizar cálculos o comparaciones, a partir de una lectura literal del gráfico estadístico. Por ejemplo, determinar la media aritmética en base a la información disponible en el gráfico; iii) Nivel 3. Leer más allá de los datos. Conlleva el obtener algún dato o información que no se observa directamente en el gráfico y que para obtenerla es necesario realizar una interpolación 0 extrapolación de los datos. Por ejemplo, estimar las ventas de una tienda en el décimo mes, con la información representada en un diagrama de barras; y iv) Nivel 4. Leer detrás de los datos. Exige valorar críticamente los datos, el tipo de gráfico utilizado, la forma en que se han recogido y/o analizado los datos, así como las conclusiones obtenidas. Por ejemplo, analizar si las conclusiones realizadas son válidas de acuerdo con el contexto de los datos.

\section{METODOLOGÍA}

La metodología se presenta en cuatro subsecciones: en la primera se detalla el diseño del estudio; en el segundo se describe la muestra del estudio; en la tercera se especifica el instrumento aplicado; $y$, finalmente, se da cuenta de los procedimientos de análisis de la información.

\section{Diseño del estudio}

Este estudio siguió una metodología mixta (Creswell y Tashakkori, 2007) y de nivel descriptivo, porque tuvo como propósito alcanzar una especificación detallada del objeto de estudio. Desde el punto de vista de la complementariedad metodológica se utilizaron datos cualitativos y cuantitativos. La perspectiva cualitativa intervino en la interpretación de los datos, al ser utilizado, como método, el análisis de contenido (Bengtsson, 2016) para estudiar la calidad de las respuestas entregadas por los profesores y los niveles de lectura alcanzados con estas. La perspectiva cuantitativa se utilizó para determinar el porcentaje de éxito de las respuestas y de los niveles de lectura logrados, así como valorar globalmente los resultados según ítem.

\section{Participantes y contexto}

Para efectos del estudio se consideró un muestreo no probabilístico de tipo accidental. La muestra estuvo constituida por 21 profesores de Educación Primaria del sistema público de la zona central de Chile, 19 mujeres (90.5\%) y 2 hombres (9.5\%), con una experiencia docente media de 11.2 años y una desviación estándar de 4.4 años. Estos profesores estaban presentes el día de la aplicación del instrumento, de un total de 25, que participaban en un curso de perfeccionamiento, recibiendo capacitación en cuatro asignaturas fundamentales: Lenguaje, Matemática, Ciencias Sociales y Ciencias Naturales para los primeros años de Educación Primaria.

\section{Instrumento}

Posterior a la firma de los consentimientos informados, se aplicó un instrumento con tres ítems relacionados con la lectura de gráficos estadísticos (tabla 1), los que han sido validados por juicio de expertos y pilotados previos a su aplicación en estudiantes de Educación Primaria (Arteaga et al., 2020). El primer ítem abordó el cambio de registro desde un pictograma a una tabla estadística. Este ítem fue extraído del estudio de Batanero et al. (2018), que evaluaba la comprensión de gráficos estadísticos en estudiantes chilenos. El ítem 2 solicita la lectura literal de ciertos elementos de un gráfico de líneas (identificar el título, reconocer las variables representadas, leer un valor del gráfico y leer una categoría asociada a un determinado valor). Al igual que el ítem anterior, fue utilizado en el estudio de Arteaga et al. (2020, 2021). Finalmente, el ítem 3 requiere que el profesor, a partir de dos gráficos que muestran la misma información ( $\mathrm{A} \circ \mathrm{B})$, pero con diferente escala, 
identifique y seleccione aquel gráfico que a su juicio sea de conveniencia para uno de los candidatos (Pedro). La actividad es una adaptación de un estudio previo (Cavalcanti y Guimarães, 2016) y utilizada en Arteaga et al. (2021).

Tabla 1: Ítems utilizados en el estudio

Ítem 1. Completa la siguiente tabla con la información mostrada en el gráfico.

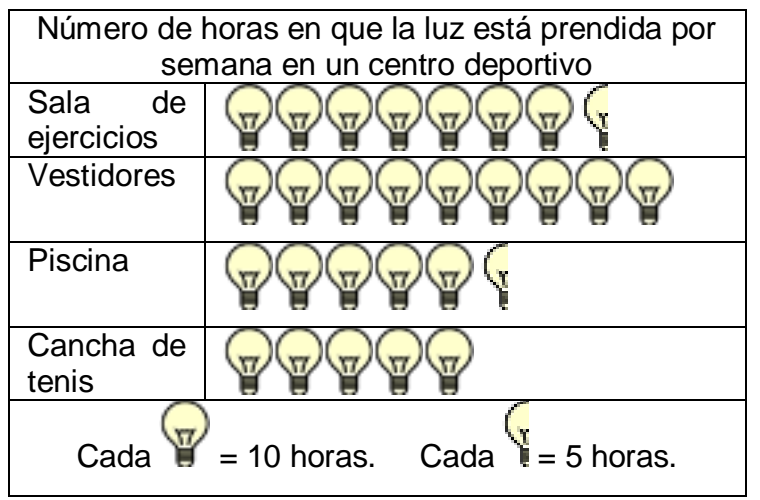

\begin{tabular}{|l|l|}
\hline \multicolumn{2}{|c|}{$\begin{array}{c}\text { Cantidad de horas por semana que está } \\
\text { prendida la luz }\end{array}$} \\
\hline \multicolumn{1}{|c|}{ Lugar } & Horas \\
\hline Sala de ejercicios & \\
\hline Vestidores & \\
\hline Piscina & \\
\hline Canchas de tenis & \\
\hline Total & \\
\hline
\end{tabular}

Ítem 2. Observa el siguiente gráfico de línea y luego responde.

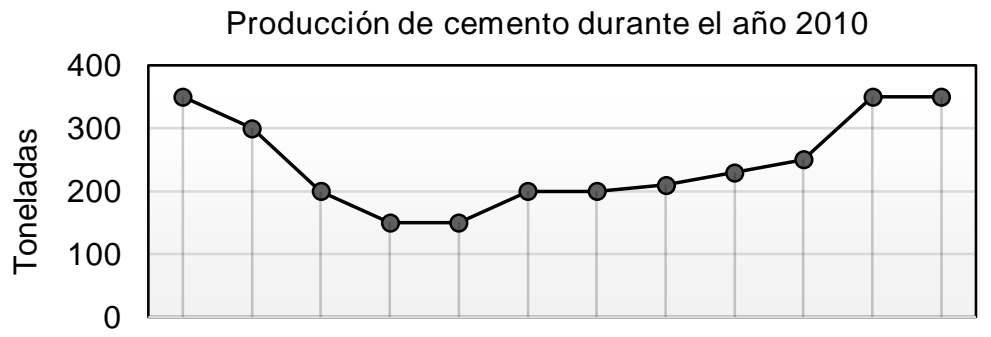

Ene Feb Mar Abr May Jun Jul Ago Sep Oct Nov Dic

Mes

1. ¿Cuál es el título del gráfico?

2. ¿Cuáles son las variables representadas?

3. ¿Cuántas toneladas de cemento se produjeron durante el mes de Febrero?

4. ¿En qué mes(es) la producción fue de 200 toneladas de cemento?

Ítem 3. Los dos gráficos muestran el mismo resultado de una encuesta electoral. ¿Cuál crees que el candidato Pedro mostraría en su campaña?

A

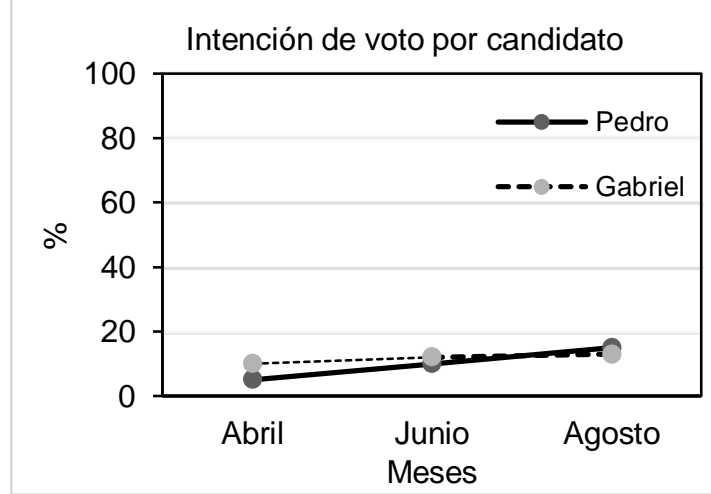

B

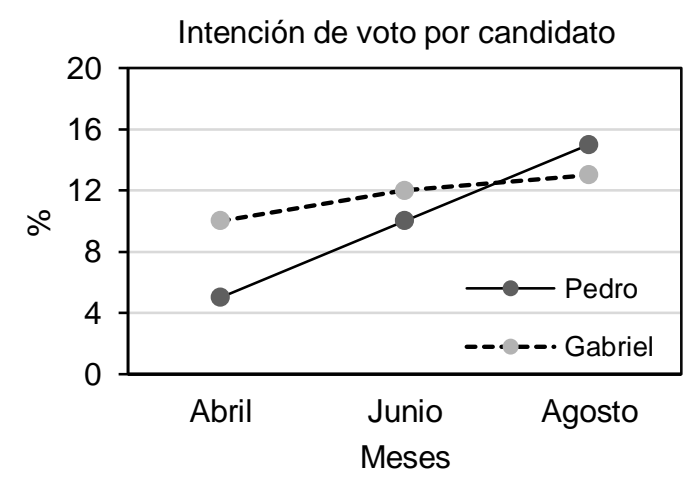

Gráfico que mostraría Pedro:

Explica tu respuesta: 


\section{Procedimiento de análisis de los datos}

En coherencia con los objetivos del estudio, los análisis consideraron los siguientes pasos: 1) analizar la calidad de las respuestas entregadas por los profesores en activo, clasificándolas en correctas, parcialmente correctas e incorrectas, estableciendo subcategorías cuando sea necesario, con el fin de caracterizar los errores que presentan; 2) establecer el nivel de lectura según la taxonomía de Curcio y cols. (Curcio, 1987; Friel et al., 2001; Shaughnessy et al., 1996), de las respuestas entregadas por los profesores. Este análisis busca evidencias que permitan reconocer un determinado nivel de lectura, teniendo como referencia el máximo que se puede alcanzar en cada ítem del instrumento: en el primer ítem se puede lograr el nivel 2 (leer dentro de los datos), en el segundo el nivel 1 (leer datos) y en el tercero el nivel 4 (leer detrás de los datos); 3) valorar globalmente las respuestas entregadas por los profesores, considerando la calidad y el nivel de lectura alcanzado, para comparar el rendimiento en cada ítem.

\section{RESULTADOS}

Para describir los resultados de cada ítem se consideraron dos aspectos: la calidad de la respuesta y el nivel de lectura que se logra. Primero, se presentan los resultados del análisis de cada ítem por separado y se finaliza con una valoración global de los resultados. Cabe destacar que para ejemplificar las respuestas se utiliza el código $\mathrm{PeA} X$, donde PeA significa profesores en activo y $X$ el número asignado al orden en que se revisó cada instrumento. Por ejemplo, PeA17 corresponde a la respuesta entregada en el instrumento del profesor en activo que se ubica en el lugar 17.

\section{Ítem 1. Calidad de lectura de un pictograma y construcción de una tabla}

Según la calidad de las respuestas entregadas por los profesores de educación primaria consultados, se han generado las siguientes categorías: tabla correcta y tabla parcialmente correcta

Tabla correcta. Se lee y se traduce correctamente todos los datos del pictograma a la tabla. Además, calcula correctamente el total. Por ejemplo, la respuesta del PeA4 que se muestra en la tabla 2.

Tabla 2: Respuesta correcta (PeA4)

\begin{tabular}{|l|c|}
\hline \multicolumn{2}{|c|}{ Cantidad de horas por semana que está prendida la luz } \\
\hline \multicolumn{1}{|c|}{ Lugar } & Horas \\
\hline Sala de ejercicios & 75 \\
\hline Vestidores & 90 \\
\hline Piscina & 55 \\
\hline Canchas de tenis & 50 \\
\hline Total & 270 \\
\hline
\end{tabular}

Tabla parcialmente correcta. Se traduce correctamente gran parte de la información, pero se omite algún valor o se realiza erradamente algún cálculo. Dentro de este análisis se han identificado dos subcategorías: $P 1$. Error en la cuantificación de iconos y P2. Error en calcular el total de la tabla. El código P, significa respuesta parcialmente correcta.

P1. Error en la cuantificación de iconos. Se cuenta un icono más/menos al leer el pictograma, lo que afecta tanto al valor asociado a la categoría como al total de la tabla. Por ejemplo, PeA11 (tabla 3) cuenta un icono más en los vestidores, obteniendo 10 y no 9 , por lo que registra 100 horas de consumo en este lugar, afectando la suma total de consumo.

Tabla 3: Respuesta con error en la cuantificación de iconos (PeA11)

\begin{tabular}{|l|c|}
\hline \multicolumn{1}{|c|}{ Cantidad de horas por semana que está prendida la luz } \\
\hline \multicolumn{1}{|c|}{ Lugar } & Horas \\
\hline Sala de ejercicios & 75 \\
\hline Vestidores & 100 \\
\hline Piscina & 55 \\
\hline Canchas de tenis & 50 \\
\hline Total & 280 \\
\hline
\end{tabular}

P2. Error en calcular el total de la tabla. Cuando se lee el pictograma y se completan las filas de la tabla correctamente, pero no calcula la suma total (PeA1) o lo hace de forma errada (PeA17), como se observa en la tabla 4. Pese a ello, en ambos casos, se evidencia la capacidad para leer un pictograma y construir una tabla. 
Tabla 4: Respuesta con error en la cuantificación de iconos (PeA11)

\begin{tabular}{|c|c|c|c|}
\hline \multicolumn{2}{|c|}{$\begin{array}{l}\text { Cantidad de horas por semana } \\
\text { que está prendida la luz }\end{array}$} & \multicolumn{2}{|c|}{$\begin{array}{l}\text { Cantidad de horas por semana } \\
\text { que está prendida la luz }\end{array}$} \\
\hline Lugar & Horas & Lugar & Horas \\
\hline Sala de ejercicios & 75 & Sala de ejercicios & 75 \\
\hline Vestidores & 90 & Vestidores & 90 \\
\hline Piscina & 55 & Piscina & 55 \\
\hline Canchas de tenis & 50 & Canchas de tenis & 50 \\
\hline Total & & Total & 275 \\
\hline \multicolumn{2}{|c|}{$\mathrm{PeA} 1$} & \multicolumn{2}{|c|}{ PeA17 } \\
\hline
\end{tabular}

En la tabla 5 se observa la distribución del tipo de respuesta entregada por los profesores en activo, según la calidad de la construcción. En ella, el $85.7 \%$ realiza correctamente la traducción, mientras que tan solo el $14.3 \%$ de los profesores realiza una construcción parciamente correcta, al contabilizar mal la cantidad de iconos $(4.8 \%)$ o equivocarse en el cálculo del total de la tabla $(9.5 \%)$.

Tabla 5: Frecuencia (y porcentaje) de tipo de respuesta según la calidad de la construcción de una tabla

\begin{tabular}{|c|c|c|}
\hline \multicolumn{2}{|c|}{ Tipo de respuesta } & Frecuencia (Porcentaje) \\
\hline \multicolumn{2}{|c|}{ Correcta } & $18(85.7)$ \\
\hline \multirow{2}{*}{ Parcialmente correcta: } & Error en la cuantificación de iconos & $1(4.8)$ \\
\cline { 2 - 3 } & Error en calcular el total de la tabla & $2(9.5)$ \\
\hline & Total & $21(100)$ \\
\hline
\end{tabular}

\section{Ítem 1. Nivel de lectura}

En segundo lugar, se analizan las respuestas de los profesores en activo según el nivel de lectura que alcanzan, según Curcio y cols. (Curcio, 1987; Friel et al., 2001; Shaughnessy et al., 1996). Las respuestas de los participantes se han clasificado, en su totalidad, en el Nivel 2. Leer dentro de los datos, implicando la lectura correcta de la cantidad de iconos en cada categoría y multiplicando según su valor, para posteriormente registrar en la tabla y calcular el total. En algunos casos, pese a evidenciar algunos problemas de lectura de cantidad o del cálculo del total, dan cuenta de su capacidad para realizar operaciones aritméticas con los datos proporcionados. Las Figuras 4, 5 y 6 corresponden a ejemplos de este nivel de lectura.

\section{Ítem 2. Calidad de lectura: lectura literal de un gráfico de líneas}

De modo similar, en el año 2010. Esta tarea conlleva la habilidad del profesor para reconocer el contexto de la información representada, dando ítem 2, se analiza la calidad de la respuesta que entregan los profesores de Educación Primaria frente a las cuatro tareas de lectura directa de ciertos elementos del gráfico de líneas. La primera tarea propuesta a los profesores consiste en identificar el título de gráfico estadístico, que informa la producción de cemento durante el sentido a los datos. El análisis de las respuestas ha permitido identificar las categorías lectura correcta, lectura parcialmente correcta y lectura incorrecta, con su respectiva descripción y ejemplo, que se muestran en la tabla 6.

Tabla 6: Calidad, descripción y ejemplo de respuesta en la lectura del título de un gráfico de líneas

\begin{tabular}{|l|l|l|}
\hline \multicolumn{1}{|c|}{ Calidad de la respuesta } & \multicolumn{1}{|c|}{ Descripción } & \multicolumn{1}{c|}{ Ejemplo de respuesta } \\
\hline Lectura correcta & $\begin{array}{l}\text { Cuando el profesor es capaz de leer } \\
\text { y registrar literalmente el título del } \\
\text { gráfico }\end{array}$ & $\begin{array}{l}\text { Producción de cemento durante el } \\
\text { año 2010 (PeA7) }\end{array}$ \\
\hline Lectura parcialmente correcta & $\begin{array}{l}\text { Cuando el PeA omite alguna palabra, } \\
\text { pero mantiene la idea central del } \\
\text { gráfico estadístico }\end{array}$ & $\begin{array}{l}\text { Producción de cemento durante el } \\
\text { año (PeA6) }\end{array}$ \\
\hline Lectura incorrecta & $\begin{array}{l}\text { Cuando se hace mención a algún } \\
\text { aspecto no presente en el título del } \\
\text { gráfico estadístico. }\end{array}$ & $\begin{array}{l}\text { Producción cemento año 2020 } \\
\text { (PeA3) }\end{array}$ \\
\hline
\end{tabular}

En la tabla 7 se muestran los tipos de respuestas y sus descripciones con respecto a la segunda tarea del ítem, generadas por la calidad de las mismas, así como ejemplos que permiten ilustrar de mejor forma las categorías lectura correcta, lectura parcialmente correcta y lectura incorrecta. 
Tabla 7: Calidad, descripción y ejemplo de respuesta en la lectura de las variables representadas en un gráfico de líneas

\begin{tabular}{|l|l|l|}
\hline \multicolumn{1}{|c|}{ Calidad de la respuesta } & \multicolumn{1}{|c|}{ Descripción } & \multicolumn{1}{c|}{ Ejemplo de respuesta } \\
\hline Lectura correcta & $\begin{array}{l}\text { Cuando se identifican y registran los variables } \\
\text { que intervienen en el gráfico de líneas. }\end{array}$ & Toneladas y meses (PeA6) \\
\hline Lectura parcialmente correcta & $\begin{array}{l}\text { Cuando se menciona una de las dos variables } \\
\text { representadas en el gráfico. }\end{array}$ & Toneladas (PeA14) \\
\hline Lectura incorrecta & $\begin{array}{l}\text { Cuando se mencionan aspectos no } \\
\text { relacionados con las variables representadas } \\
\text { en el gráfico }\end{array}$ & 12 (PeA17) \\
\hline
\end{tabular}

Con respecto a la tercera tarea del ítem 2, en la tabla 8 se muestran las categorías de respuesta, con sus respectivas descripciones y ejemplos. En ella se puede observar que las respuestas se han podido clasificar, según su calidad, en correctas y parcialmente correctas, evidenciando la facilidad de esta tarea para los profesores.

Tabla 8: Calidad, descripción y ejemplo de respuesta en la lectura de las variables representadas en un gráfico de líneas

\begin{tabular}{|l|l|l|}
\hline \multicolumn{1}{|c|}{ Calidad de la respuesta } & \multicolumn{1}{|c|}{ Descripción } & \multicolumn{1}{c|}{ Ejemplo de respuesta } \\
\hline Lectura correcta & $\begin{array}{l}\text { Cuando se es capaz de leer y registrar que la } \\
\text { producción de cemento durante febrero fue de } \\
300 \text { toneladas }\end{array}$ & 300 toneladas" (PeA7) \\
\hline Lectura parcialmente correcta & $\begin{array}{l}\text { Cuando la respuesta presenta alguna } \\
\text { imprecisión, es decir, no mencionan la unidad } \\
\text { de medida utilizada (toneladas) }\end{array}$ & 300 (PeA13) \\
\hline
\end{tabular}

La cuarta tarea exige realizar una lectura inversa, es decir, se deben encontrar los meses en que la producción es de 200 toneladas. Dado que la respuesta conlleva identificar tres meses (marzo, junio y julio), las categorías se han definido de acuerdo con la cantidad de meses señalados, como se describe en la tabla 9.

Tabla 9: Calidad, descripción y ejemplo de respuesta en la lectura de las variables representadas en un gráfico de líneas

\begin{tabular}{|l|l|l|}
\hline \multicolumn{1}{|c|}{ Calidad de la respuesta } & \multicolumn{1}{|c|}{ Descripción } & \multicolumn{1}{|c|}{ Ejemplo de respuesta } \\
\hline Lectura correcta & $\begin{array}{l}\text { Cuando se identifican y registran los meses en } \\
\text { que la producción de cemento es de 200 } \\
\text { toneladas }\end{array}$ & Marzo, junio y julio (PeA9) \\
\hline Lectura parcialmente correcta & $\begin{array}{l}\text { Cuando se menciona uno o dos meses en que } \\
\text { la producción de cemento fue de 200 } \\
\text { toneladas. Además, se incluyen aquellos que } \\
\text { presente imprecisión en algún mes. }\end{array}$ & Marzo y mayo (PeA20) \\
\hline Lectura incorrecta & $\begin{array}{l}\text { Cuando no se menciona ninguno de los meses } \\
\text { en que la producción de cemento es de 200 } \\
\text { toneladas. Evidenciando el no tener la } \\
\text { capacidad para leer literalmente un gráfico de } \\
\text { líneas. }\end{array}$ & $\begin{array}{l}\text { Enero, febrero, octubre, } \\
\text { noviembre y diciembre }\end{array}$ \\
\hline
\end{tabular}

En la tabla 10 se presenta el tipo de respuesta para las cuatro tareas del ítem 2. En ella, vemos que la primera tarea, en la que se pide identificar el título del gráfico de líneas, es la que presenta menor dificultad para los profesores $(85.7 \%)$, seguida de la tercera tarea, que pide leer la producción de cemento en el mes de febrero (76.2\%), esta última es la única que no presenta respuestas incorrectas. Por otro lado, la cuarta tarea en la que se pide encontrar los tres meses en que la producción de cemento es de 200 toneladas $(61.9 \%)$ es la que presenta mayor complejidad.

Tabla 10: Frecuencia (y porcentaje) de tipo de respuesta según la calidad de la lectura

\begin{tabular}{|c|c|c|c|c|}
\hline Tipo de respuesta & 2.1 & 2.2 & 2.3 & 2.4 \\
\hline Correcta & $18(85.7)$ & $14(66.7)$ & $16(76.2)$ & $13(61.9)$ \\
\hline Parcial & $2(9.5)$ & $5(23.8)$ & $5(23.8)$ & $6(28.6)$ \\
\hline Incorrecta & $1(4.8)$ & $2(9.5)$ & $0(0)$ & $2(9.5)$ \\
\hline
\end{tabular}


Seguidamente analizamos los niveles de lectura de Curcio y cols. (Curcio, 1987; Friel et al., 2001; Shaughnessy et al., 1996) que alcanzan los profesores de acuerdo con sus respuestas. Estas se pueden clasificar en los siguientes niveles: i) Nivel 0 . Cuando presenta dificultades para leer el título del gráfico, proporcionando información parcial o errónea. La única respuesta considerada dentro de este nivel es la entregada por el $\mathrm{PeA} 3$, quien omite palabras y cambia el año registrado en el título del gráfico: "Producción cemento año 2020" (PeA3); ii) Nivel 1. Leer los datos. Cuando no presenta dificultades para leer el título del gráfico o su respuesta se basa en la lectura literal de un elemento del gráfico estadístico. En este caso, se incluyen algunas respuestas consideradas incorrectas, pero que se extraen directamente de la representación. Por ejemplo, la respuesta de PeA15: "300 toneladas". En la tabla 11 se presenta la distribución de los niveles de lectura alcanzado por las respuestas de los profesores a las cuatro tareas propuestas en el ítem 2. En ella, se observa que casi la totalidad de los participantes es capaz de leer literalmente diversos elementos presentes en el gráfico de líneas.

Tabla 11: Frecuencia (y porcentaje) del tipo de respuesta obtenidas en el ítem 2

\begin{tabular}{|c|c|c|c|c|}
\hline Nivel de lectura & 2.1 & 2.2 & 2.3 & 2.4 \\
\hline 1 & $20(93.2)$ & $21(100)$ & $21(100)$ & $21(100)$ \\
\hline 0 & $1(4.8)$ & $0(0)$ & $0(0)$ & $0(0)$ \\
\hline
\end{tabular}

\section{Ítem 3. Calidad de lectura: Selección de gráfico estadístico}

En este ítem se presentan dos gráficos con la misma información, y los profesores deben identificar y seleccionar el que usaría Pedro para presentar durante su campaña. Para ello, en primer lugar, se analiza el gráfico que, según los profesores, debería mostrar. Al analizar las respuestas proporcionadas por los participantes vemos que $17(81 \%)$ de ellos indican correctamente el gráfico que usaría Pedro en su campaña. Por el contrario, 4(19\%) profesores indican el gráfico que no conviene utilizar.

\section{Ítem 3. Nivel de lectura}

Seguidamente, se analizan los argumentos que declaran los profesores para justificar la elección del gráfico que, a su juicio, Pedro debería usar en su campaña. En este caso, se exige un nivel de lectura 4 (leer detrás de los datos) (Curcio, 1987; Friel et al., 2001; Shaughnessy et al., 1996), porque deben analizar críticamente el gráfico, en particular, cuestionar al efecto visual que provoca la modificación de la escala.

Las respuestas de los profesores se han clasificado en los siguientes niveles: i) Nivel 0. Cuando no explica la elección del gráfico estadístico o su elección es errada, sin observar la tendencia o leer algún valor específico de este. Ejemplo de esta situación es el PeA8, quien selecciona el gráfico A y no justifica dicha elección; ii) Nivel 1. Leer los datos. Cuando lee directamente un dato o algunos de ellos, sin interpretar ni realizar cálculos. No se observan respuestas asociadas a este nivel; iii) Nivel 2. Leer dentro de los datos. Considera realizar, luego de una lectura literal, cálculos, comparaciones o relaciones entre los datos. Es el caso de la respuesta que entrega el PeA1, al mencionar la tendencia al alza de los datos y comparar ambas líneas: "Porque Pedro estaría con tendencia al alza y superaría al otro candidato" (PeA1); iv) Nivel 3. Leer más allá de los datos. Realizar inferencias o predicciones con la información presentada en el gráfico estadístico. Este nivel no se ha observado en las respuestas de los profesores; v) Nivel 4. Leer detrás de los datos.

Cuando se realiza una valoración crítica de la representación, de las conclusiones obtenidas y/o forma de recolección de datos. En este caso, los profesores en activo, que seleccionan el gráfico correcto (B), asumen que los gráficos muestran la misma información y mencionan explícitamente la modificación de la escala y el impacto visual que esto produce. Se observa que los argumentos presentan ciertas imprecisiones. Ejemplo de este nivel es la respuesta del PeA21, al mencionar que con una menor escala (2 unidades) se observa con mayor claridad la intención de voto a favor de Pedro sobre Gabriel: "Porque en el gráfico B se evidencia una diferencia en la comparación de la intención de voto por candidato ya que la escala utilizada al ser de 2 en 2 permite que se vea la diferencia a simple viste" (PeA21).

La tabla 12 presenta la distribución de los niveles de lectura que alcanzaron las respuestas de los profesores en este ítem. En ella observamos que tan solo tres profesores (14.3\%) logran el nivel más alto exigido (leer detrás de los datos). La mayoría de los participantes alcanzan el nivel de leer dentro de los datos (76.2\%), las que se basan en la descripción de tendencias, comparación de valores, percepción de intención de voto, etc., pero sin llegar a realizar una lectura crítica. 
Tabla 12: Frecuencia y porcentaje de los niveles de lectura alcanzados por los profesores

\begin{tabular}{|c|c|c|}
\hline Nivel de lectura & Frecuencia & Porcentaje \\
\hline 0 & 2 & 9.5 \\
\hline 2 & 16 & 76.2 \\
\hline 4 & 3 & 14.3 \\
\hline
\end{tabular}

\section{Análisis general de los datos}

Para realizar una valoración general de las respuestas entregadas por los profesores a cada ítem, se considera la calidad y el nivel de lectura evidenciados, según se detalla en la tabla 13. En el ítem 1, se puede alcanzar un máximo de 4 puntos, 2 por la calidad de su respuesta (2: correcta; 1: parcialmente correcta, 0 : incorrecta) y 2 según el nivel de lectura alcanzado. En el ítem 2, el puntaje máximo posible de obtener es 12 , tres por cada una de las tareas propuestas (4), es decir, 2 por la calidad de la respuesta (2: correcta; 1 : parcialmente correcta, 0 : incorrecta) y 1 según el nivel de lectura (0: lectura incorrecta o no lectura, 1: lectura literal). El máximo puntaje posible de alcanzar en el ítem 3 es de $5 ; 0$ a 1 (incorrecto o correcto, respectivamente) por la selección del gráfico y 4 por el nivel de lectura alcanzado.

Tabla 13: valoración general de los ítems

\begin{tabular}{|c|c|c|c|c|}
\hline Ítem & Tareas & Calidad de la respuesta & Nivel de lectura & Puntaje máximo \\
\hline 1 & 1 & 0 a 2 puntos & 0 a 2 puntos & 4 \\
\hline 2 & 4 & 0 a 2 puntos (por tarea) & 0 a 1 puntos & 12 \\
\hline 3 & 1 & 0 a 1 puntos & 0 a 1 puntos & 5 \\
\hline
\end{tabular}

Posteriormente, para comparar los puntajes alcanzados en los tres ítems, se establecen intervalos entre 0 y 1 , dividiendo los puntajes logrados por el máximo teórico (4, 12 y 5, respectivamente). Con ello, podemos observar la media del nivel de logro: 0.96 ítem 1; 0.88 ítem 2 y 0.58 ítem 3. De acuerdo con lo anterior, el ítem 1 es el más sencillo para los profesores en activo y el ítem 3 presenta mayores dificultades. Estos resultados son confirmados en los box-plot de la figura 1, donde las puntuaciones alcanzadas por los profesores en el ítem 1 son más altos que los restantes, debido a que, la mediana y los cuartiles 1 y 3 son iguales a 1 . Por el contrario, la menor puntuación se logra en el ítem 3, donde los cuartiles 1, 2 (mediana) y 3 son iguales a 0.6, lo que muestra poca variación de la distribución de los datos, siendo el participante PeA8 quien presenta la única respuesta valorada con 0 puntos. Las puntuaciones del ítem muestran una mayor dispersión de los datos, donde el cuartil 1 es 0.83 , la mediana es 0.92 y el cuartil 3 es 1 , presentando un valor atípico (la puntación de PeA3 es 0.5).

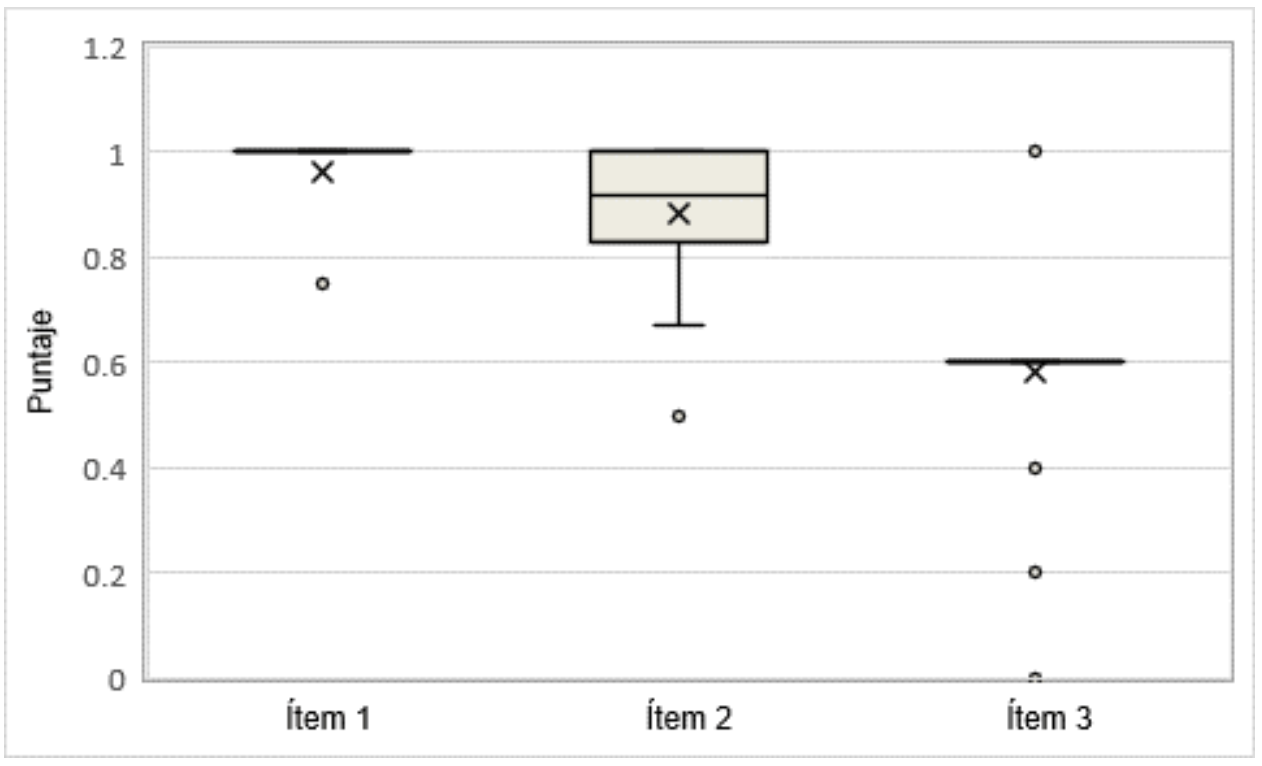

Fig. 1: Distribución de las puntuaciones globales 


\section{DISCUSIÓN}

Analizar el conocimiento disciplinario y didáctico sobre elementos estadísticos que presentan profesores en activo es fundamental para determinar las dificultades que se pueden presentar en los procesos de enseñanza y de aprendizaje; así como conjeturar sobre las temáticas que los profesores tenderían a no tratar o hacerlo superficialmente, porque no se puede enseñar lo que no se conoce. En este caso, nuestro interés recae en indagar sobre la calidad y el nivel de lectura sobre gráficos estadísticos logrado por profesores de Educación Primaria en activo, que participan en un curso de perfeccionamiento. En concreto, se plantea un instrumento con tres ítems, el primero requiere traducir la información de un pictograma a una tabla estadística, el segundo conlleva leer algunos elementos presentes en un gráfico de líneas, y el tercero exige justificar la elección de un gráfico; movilizando los niveles de lectura 2, 1 y 4 de Curcio y cols. (Curcio, 1987; Friel et al., 2001; Shaughnessy et al., 1996), respectivamente.

Respecto de los resultados del ítem 1, podemos observar que la mayoría de los profesores no presentan dificultades en completar la tarea $(85.7 \%$ correctamente y $14.3 \%$ parcialmente correcta), es decir, son capaces de leer correctamente un pictograma con icono no unitario y completar una tabla de frecuencias, realizando las operaciones pertinentes y calculando el total asociado. Al considerar que esta actividad demanda un nivel 2 de lectura, se puede observar que los resultados del presente estudio son mejores que los informados en Rodríguez-Alveal y Sandoval (2012), al trabajar con gráficos de barras (simples y dobles) y sectores, donde este nivel es alcanzado en un porcentaje inferior al 20\%. En los resultados de este estudio, pese a ser positivos, llama la atención el tipo de errores que cometen algunos profesores (cuantificación y cálculo), dado que el pictograma está explícitamente indicado para ser trabajado desde el primer año de Educación Primaria (MINEDUC, 2018), y ampliamente presente en los libros de texto (Díaz-Levicoy et al., 2016).

El ítem 2 estuvo conformado por cuatro tareas, que exigen la lectura literal de la información presente en el gráfico de líneas (nivel 1 de lectura). El $85.7 \%$ de los profesores puede leer y registrar correctamente el título del gráfico; el $66.7 \%$ es capaz de identificar y registrar las variables representadas en el gráfico de líneas; el $76.2 \%$ puede leer la producción durante un determinado mes; y $61.9 \%$ logra identificar los meses en que se alcanzó una determinada producción. Estos resultados son menores a los alcanzado por profesores en formación y en activo, reportados por Rodríguez-Alveal y Sandoval (2012), donde las respuestas correctas fluctúan entre un $93 \%$ y $100 \%$. Estos resultados podrían ser similares si consideramos aquellos profesores en que sus respuestas se han categorizado como parcialmente correcta, es decir, donde se han omitido palabras del título, pero no afecta la información que se representa (tarea 1), registrar solo una de las variables que intervienen en el gráfico (tarea 2), no utilizar la unidad de medida (tarea 3) y registrar algunos de los meses en que la producción fue la solicitada (tarea 4). Estos resultados son preocupantes, considerando que se aplicó a profesores en activo y por el nivel de complejidad, ya que es considerada una tarea sencilla (nivel 1 de lectura), pues solo se espera que reconozcan los elementos clave de esta representación (elementos constituyentes).

En el ítem 3, donde se exige un nivel de lectura 4 de Curcio y cols. (Curcio, 1987; Friel et al., 2001; Shaughnessy et al., 1996), el $81 \%$ de los profesores selecciona correctamente el gráfico de la situación, pero solo el $14.3 \%$ son capaces de realizar una justificación adecuada de dicha elección, es decir, menciona el efecto visual que provoca modificar la escala de un gráfico. Las respuestas de nivel 2 de Curcio y cols. reúne el $76.2 \%$ de los profesores, es decir, observan la variación de los datos en el gráfico, pero no mencionan el impacto que tiene en la gráfica la modificación de la escala. Por lo anterior, este es el ítem que presenta mayor dificultad para los profesores en activo, coincidiendo con lo observado en las investigaciones previas con niños y adultos en que se ha exigido un nivel de lectura 4 (Cavalcanti y Guimarães, 2016; Arteaga et al., 2021).

Al comparar el rendimiento de los profesores de Educación Primaria en activo por ítem, por medio de las puntuaciones totales (considerando la calidad de la respuesta y el nivel de lectura alcanzado), en una escala de 0 a 1, vemos que el ítem 1 presentó mayor facilidad (0.96), a diferencia del tercero que resultó ser más complejo (0.58). Por su parte, el ítem 2 presenta menor nivel de logro que el ítem 1, pese a exigir un mayor nivel de lectura, por lo que es probable que el tipo de representación tenga mayor influencia que el nivel de lectura exigido.

\section{CONCLUSIONES}

A partir de los resultados obtenidos, se pueden extraer las siguientes conclusiones: i) Los profesores no presentan mayores dificultades para traducir información desde un pictograma con icono no unitario a una tabla estadística; ii) El estudio muestra que algunos los profesores en activo tienen dificultades para identificar elementos estructurales de un gráfico de líneas; iii) La mayoría de los profesores consultados tienen dificultades para justificar el impacto visual que produce la modificación de la escala en un gráfico de líneas. 


\section{REFERENCIAS}

Arteaga, P., Batanero, C., Contreras, J.M. y Cañadas, G.R., Evaluación de errores en la construcción de gráficos estadísticos elementales por futuros profesores, https://doi.org/10.12802/relime.13.1911, Revista Latinoamericana de Investigación en Matemática Educativa, 19(1), 15-40 (2016)

Arteaga, P., Díaz-Levicoy, D. y Batanero, C., Assessing Chilean primary school children's understanding of statistical graphs, https://doi.org/10.17648/acta.scientiae.5884, Acta Scientiae, 22(2), 2-24 (2020)

Arteaga, P., Díaz-Levicoy, D. y Batanero, C., Primary school students' reading levels of line graphs, Statistics Education Research Journal, en prensa, (2021)

Batanero, C., Díaz-Levicoy, D. y Arteaga, P., Evaluación del nivel de lectura y la traducción de pictogramas por estudiantes chilenos de Educación Básica, https://doi.org/10.35763/aiem.v0i14.231, Avances de Investigación en Educación Matemática, 14, 49-64 (2018)

Bengtsson, M., How to plan and perform a qualitative study using content analysis, https://doi.org/10.1016/j.npls.2016.01.001, NursingPlus Open, 2, 8-14 (2016)

Callingham, R. y Watson. J.M., The development of statistical literacy at school, Statistics Education Research Journal, 16(1), 181-201 (2017)

Cavalcanti, M. y Guimarães, G., Compreensão de adultos e crianças sobre escala representada em gráficos, Perspectivas da Educação Matemática, 9(21) 849-868 (2016)

Curcio, F.R., Comprehension of mathematical relationships expressed in graphs, https://doi.org/10.2307/749086, Journal for Research in Mathematics Education, 18(5), 382-393 (1987)

Creswell, J.W. y Tashakkori, A., Developing publishable mixed methods manuscripts, https://doi.org/10.1177/1558689806298644, Journal of Mixed Methods Research, 1(2), 107-111 (2007)

del Pino, G. y Estrella, S., Educación estadística: relaciones con la matemática, Pensamiento Educativo. Revista de Investigación Educacional Latinoamericana, 49(1), 53-64 (2012)

Díaz-Levicoy, D., Batanero, C., Arteaga, P. y Gea, M.M., Chilean children's reading levels of statistical graphs, https://doi.org/10.29333/iejme/5786, International Electronic Journal of Mathematics Education, 14(3), $689-700$ (2019)

Díaz-Levicoy, D., Batanero, C., Arteaga, P. y Gea, M.M., Gráficos estadísticos en libros de texto de Educación Primaria: Un estudio comparativo entre y Chile, https://dx.doi.org/10.1590/1980-4415v30n55a20, Boletim de Educação Matemática, 30(55), 713-737 (2016)

Espinel, C., Bruno, A. y Plasencia, I., Statistical graphs in the training of teachers, Joint ICMI/IASE study: teaching statistics in school mathematics. Challenges for teaching and teacher education, G. Burrill, C. Reading y A. Rossman, ICMI e IASE, pp 1-6, Monterrey, México (2008)

Estrella, S., Olfos, R. y Mena-Lorca, A., Pedagogical knowledge of statistics content among primary school teachers, https://doi.org/10.1590/s1517-97022015041858, Educacao e Pesquisa, 41(2), 477-493 (2015)

Friel, S., Curcio, F. y Bright, G., Making sense of graphs: critical factors influencing comprehension and instructional implications, Journal for Research in mathematics Education, 32(2), 124-158 (2001)

Gal, I., Adult's statistical literacy: Meaning, components, responsibilities, International Statistical Review, 70(1), 1-25 (2002)

Gal, I., Teaching for statistical literacy and services of statistics agencies, The American Statistician, 57(2), 80-84 (2003)

Gal, I., Does CensusAtSchool develop statistical literacy?, https://doi.org/10.3233/SJI-2011-0737, Statistical Journal of the IAOS, 27(3-4), 229-230 (2011).

Jurečková, M. y Csachová, L., Statistical literacy of Slovak lower secondary school students, Technium Social Sciences Journal, 9, 163-173 (2020)

Koetsenruijter, A.W.M., Using numbers in news increases story credibility, https://doi.org/10.1177/073953291103200207, Newspaper Research Journal, 32(2), 74-82 (2011)

McConway, K., Statistics and the media: a statistician's view, https://doi.org/10.1177/1464884915593243, Journalism, 17(1), 49-65 (2016)

MINEDUC, Bases curriculares. Primero a sexto básico, Unidad de Currículum y Evaluación, Santiago, Chile (2018)

MINEDUC, Estándares orientadores para egresados de carreras de Pedagogía en Educación Básica. Estándares pedagógicos y disciplinarios, LOM, Santiago, Chile (2012)

Monteiro, C. y Ainley, J., Investigating the interpretation of media graphs among student teachers, International Electronic Journal of Mathematics Education, 2(3), 188-207 (2007)

Rodríguez-Alveal, F. y Díaz-Levicoy, D., Avaliação da alfabetização gráfica de professores da Educação Básica em formação e em exercício, https://doi.org/10.1590/0104-4060.68977, Educar em Revista, 35(78), 85-103 (2019) 
Rodríguez-Alveal, F. y Sandoval, P., Habilidades de codificación y descodificación de tablas y gráficos estadísticos: un estudio comparativo en profesores y alumnos de pedagogía en enseñanza básica, https://doi.org/10.1590/S141440772012000100011, Avaliação: Revista da Avaliação da Educação Superior, 17(1), 207-235 (2012)

Shaughnessy, J.M., Garfield, J. y Greer, B., Data handling, International handbook of mathematics education, A.J. Bishop, K. Clements y otros 3 editores, Kluwer Academic Publishers, pp 205-237, Dordrecht, Holanda (1996) 